\title{
Modelling and numerical simulation of the transport phenomena in water thermal energy storage tanks
}

\author{
Nikola Kaloyanov ${ }^{1}$, Rosen Tsecov ${ }^{1}$ and Nina Penkova ${ }^{2, *}$ \\ ${ }^{1}$ Technical university of Sofia, Department of Thermal and Refrigeration Engineering, Blvd. Sv. \\ Kliment Ohridski 8, 1756 Sofia, Bulgaria \\ ${ }^{2}$ University of chemical technology and metallurgy, Department of Silicate Technology, Blvd. Sv. \\ Kliment Ohridski 8, 1756 Sofia, Bulgaria
}

\begin{abstract}
Mathematical model for numerical simulation of the transient heat transfer and fluid flows in water thermal energy storage tanks is developed. The model allows analysis of the thermal fields in the accumulators at different schemes and modes of charging and discharging. It was verified and validated based on experimentally obtained information about the temperature stratification at charging of a thermal accumulator at a laboratory solar system. The proposed approach for numerical study of the thermal energy storage is convenient for parametrical estimation and improvement of the efficiency of the thermal systems.
\end{abstract}

\section{Introduction}

The thermal energy storage (TES) in the form of sensible heat in insulated water tanks is the most widely used method at systems where the periods of energy production and consumption do not coincide. The water heat accumulators are key elements for the efficiency of the solar heating systems, thermal power generation and industrial systems [1]. The temperature stratification in the liquid domain due to the buoyancy can increase the performance of these technologies:

- the upper water levels can be enough hot at lower accumulated amount of heat and the thermal loses through the tank walls are smaller in comparison to the unstratified accumulators;

- the cooler water in the bottom of the tanks leads to a higher temperature difference in the heat exchangers at the energy source and to more efficient heat transfer in them.

Many investigations aiming enhancement of the temperature stratification in water thermal accumulators are known from the literature. The first of them are based on experimental measurements, balance equations and one-dimensional analytical models of the temperature field $[2,3]$. They allow relatively accurate prediction of the temperature variation along the axis of vertical cylindrical tanks. The advanced software for computational fluid dynamics (CFD) and heat transfer (CHT) allows numerical simulation of the three-dimensional

* Corresponding author: nina@uctm.edu 
transient fields in the accumulating media in order to increase the efficiency of the thermal systems [4 - 6]. Such investigation are oriented to improvements of the accumulator shape [7], locations and geometries of the inlets and outlets [8], internal constructions for better temperature stratification [9] and charging and discharging flow parameters. The optimization of the design and the operation mode depends of the thermal systems where the thermal accumulator is integrated. These problems are not fully solved at the various technologies and applications of heat accumulators, obvious from the continuous research oriented on the topic.

The aim of the investigations in the present paper is a precise model for numerical simulation of the 3D transient fields in water tanks for TES, allowing prediction and improvement of the efficiency of thermal systems for domestic applications.

\section{Conceptions for modeling of the transient fluid flow and heat transfer in water thermal accumulators}

A three-dimensional geometrical model of the accumulator is generated, including all inlet and outlet pipes. Different scenarios of charging and discharging of the tank can be simulated accepting them as open or closed (walls). The pipe walls do not influence significantly on the processes and can be modeled as boundary interfaces [6]. The walls of the tank are also excluded from the geometrical model. The heat transfer through them can be modeled according to the boundary conditions in Table 1 . The water and pipe domains are discretized by a mesh of finite volumes in order to solve numerically the system of partial differential equations:

- continuity equation;

- momentum equations at non zero gravity to reflect the buoyancy;

- thermal energy equation;

- turbulence model.

The initial numerical solutions of the model for the thermal accumulator, described in the next part of the paper, proved the advantage of Renormalization Group (RNG) k-epsilon turbulence model - it leads to a quicker convergence than the standard one. That is in accordance to the tips for the modeling of the turbulence in [10] and the conclusions of other research of turbulence flows with swirls [11]. The influence of the buoyancy on the turbulence is taken into account using Boussinesq buoyancy model in the production and dissipation terms in the RNG k-epsilon model.

The boundary conditions are summarized in Table 1 .

Table 1. Boundary conditions.

\begin{tabular}{|l|c|}
\hline \multicolumn{1}{|c|}{ Boundary } & Variable \\
\hline Inlet of the hot flow at charging & $\begin{array}{c}\text { Velocity or mass flow and temperature variation with the } \\
\text { time. }\end{array}$ \\
\hline Inlet of the cold flow at discharging & Velocity or mass flow and temperature. \\
\hline Outlets & Mass flow or gauge pressure. \\
\hline $\begin{array}{l}\text { Tank walls (heat exchanging } \\
\text { boundaries) }\end{array}$ & $\begin{array}{c}\text { Coefficient of heat transfer from the internal surface to } \\
\text { the ambient environment: } U=\frac{1}{R_{s e}+\sum_{i=1}^{n} R_{i}}, \mathrm{Wm}^{-2} \mathrm{~K}^{-1} ; \\
\text { ambient temperature } t_{a m b}\end{array}$ \\
\hline $\begin{array}{l}\text { Pipe walls in the water domain } \\
\text { (interfaces between the tank and pipe } \\
\text { domains) }\end{array}$ & $\begin{array}{c}\text { Thermal resistance of the wall } R_{w} . \\
R_{w}=\frac{d_{e x}-d_{i n}}{2 \lambda}, \mathrm{m}^{2} \mathrm{KW}^{-1} \text { at } \frac{d e x}{d i n} \leq 2\end{array}$ \\
\hline
\end{tabular}


The variables in the equations in Table 1 are: $R_{s e}$ and $R_{i}$ - thermal resistances of the external thermal boundary layer and solid layers of the tank envelopes, $\mathrm{m}^{2} \mathrm{KW}^{-1} ; d_{e x}$ and $d_{i n}-$ external and internal diameters of the walls, $\mathrm{m} ; \lambda=$ thermal conductivity of the pipe wall, $\mathrm{Wm}^{-1} \mathrm{~K}^{-1}$.

The initial conditions include:

- initial temperature field in the water domain. It can be uniform or obtained as result of previous numerical simulation of charging or discharging of the tank;

- $\quad$ zero initial velocity and turbulence ratio;

- $\quad$ initial pressure distribution $p_{\text {in }}$ taking into account hydrostatic pressure variation in the tank. For example at vertical tank parallel on $z$ direction and $z=0$ at the bottom:

$$
p_{\text {in }}=\rho_{w} g(H-z), P a
$$

where $H=$ the vessel height, $\mathrm{m} ; \rho_{w}=$ water density, $\mathrm{kgm}^{-3} ; g=$ gravity acceleration, $\mathrm{ms}^{-2}$.

For better convergence, the transient processes are simulated numerically at time steps at which the average Courant number is smaller than one at the correspondent mesh size. As finer is the mesh as smaller are time steps.

\section{Experimental tests and numerical study of transport phenomena in a water accumulator, integrated in solar system}

\subsection{Object of investigation}

The thermal accumulator is a part of a laboratory solar installation situated on the territory of Technical University of Sofia (fig. 1). The water tank is thermally charging indirectly via heat exchanger. The internal diameter and height of the water tank (fig. 2) are correspondently $0.788 \mathrm{~m}$ and $2.114 \mathrm{~m}$. The water volume is $1000 \mathrm{l}$. The tank envelopes, made by carbon style are insulated.

Twelve thermocouples Platina 100 measure the temperatures in the tank at different levels. They are localized radially: six in the "right" side (PT100-1R - 6R) and six on the "left" side (PT100-1L - 6L). The temperatures, measured by them are written and averaged at each level. The temperatures on the inlet and the outlets are also measured by thermocouples T1, T2 and T3. The level of the inlet at the thermal charging of the accumulator can vary but it is always higher according to the outlet in order to ensure temperature stratification in the axial direction.

Different regime of charging and discharging are implemented varying the mass flows and the charging level of the supplied water flow. Detail information of the measurements at variations of the daily solar irradiation and the regime parameters is given in [12]. A part of that information for three modes of thermal charging of the water tank (Scenario 1, 2 and 3) is used at the boundary conditions for the numerical simulation of the processes and for validation of the models. Figures 3-4 show the measured temperature variations at the inlets, outlets and the different levels of the accumulator space for these scenarios. The higher temperatures in Point 1 in comparison to the bottom and outlet temperatures are probably caused by insufficient calibrations or other problems at the upper sensors. The numerical simulations, discussed in the next section showed that the temperatures in Point 1 have to be higher according to the outlet temperature. 


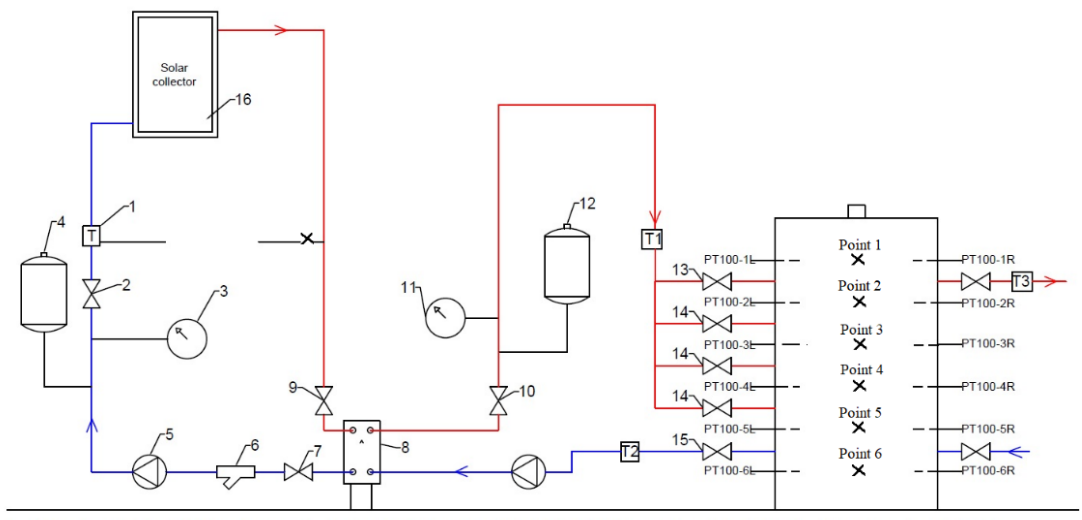

1 - Kamsturp Multical 402 heat meter; 2 - Ferro DN32R crane; 1 1/4; PN16; 3- Manometer up to 4 bar; 4 - Expansion tank Aquapress AMV40; 5 - CP Imppumps NMT PLUS 25 / 80-180; 6 - Filter; 7 - Shut-off valve DN32R; 11/4; PN16; 8 - Heat exchanger; 9 - Shut-off valve DN32R; 1 1/4; PN16; 10 - Shut-off valve DN32R; 1 1/4; PN16; 11 - Manometer up to 4 bar; 12 - Expansion vessel; 13 - DN40R; 1 1/2; PN16; 14 - DN25R; 1; PN16; 15 - DN4OR; 1 1/2; PN16; 16 - Solar collector TiSUN FM-S2.5; T1, T2 and T3 - Thermocouples; RT100-1L - 6L and RT100-1R - 6R - Thermocouples Platina 100

Fig. 1. Scheme of a solar thermal installation with water thermal energy storage tank

a)

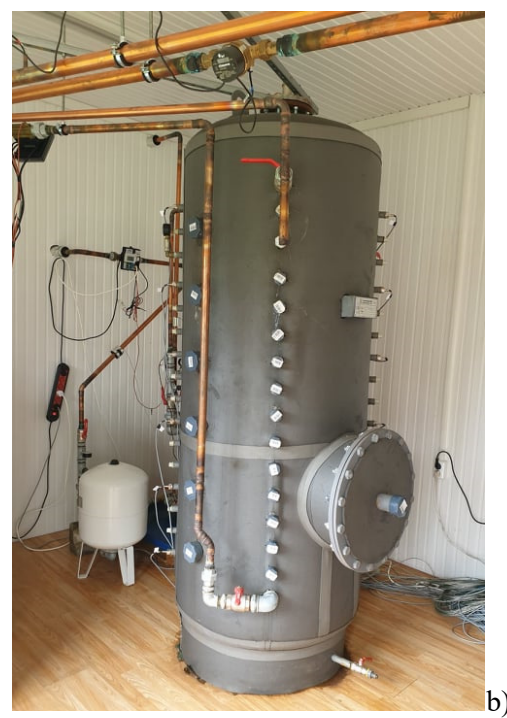

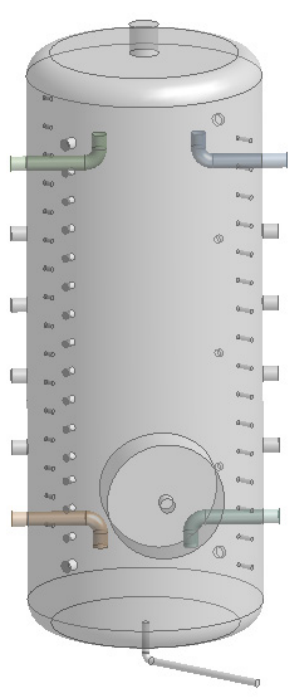

Fig. 2. Water tank for thermal energy storage (a) and its geometrical model (b) 


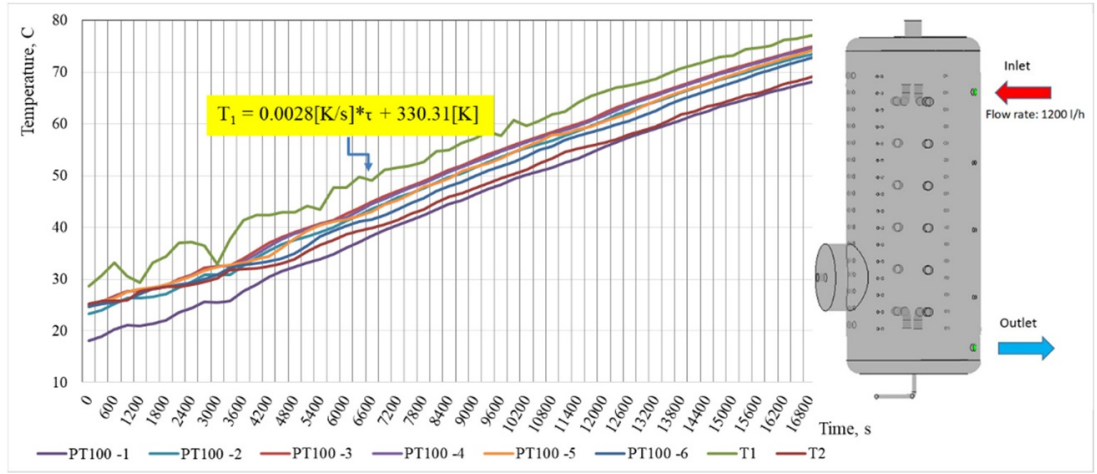

Fig. 3. Temperatures in the water tank, inlet and outlet pipes at supplied flow rate of $1200 \mathrm{l} / \mathrm{h}$ (Scenario 1)

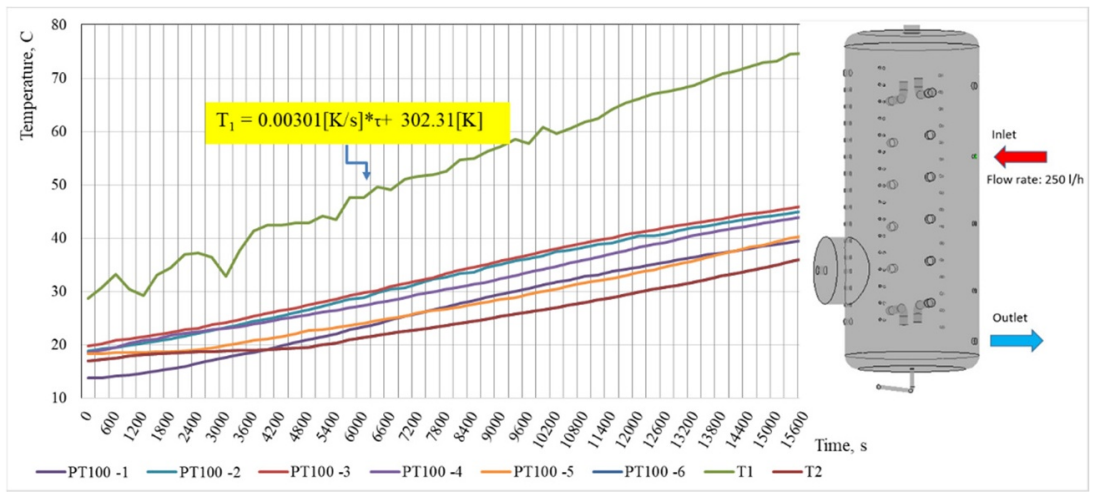

Fig. 4. Temperatures in the water tank, inlet and outlet pipes at supplied flow rate of $250 \mathrm{l} / \mathrm{h}$ (Scenario 2)

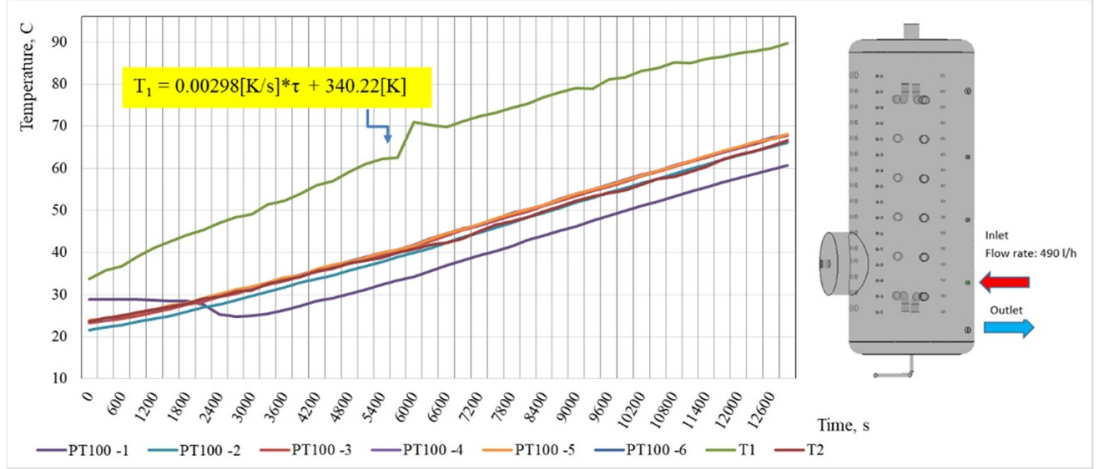

Fig. 5. Temperatures in the water tank, inlet and outlet pipes at supplied flow rate of $490 \mathrm{l} / \mathrm{h}$ (Scenario 3) 


\subsection{CFD analysis and validations of the models}

The geometrical model contains all features of the real object although some of them are not used. It is discretized by a finite volumes mesh, consisted of 302613 nodes and 1522587 elements (fig. 6). The nodes on the interfaces between the pipe and accumulator space are common for the two domains.

Three scenarios of charging are simulated numerically by ANSYS 19/CFX. The maximal time steps, allowing sufficient convergence at the transient computations are 0.6 seconds. That led to a relatively long computational time. So parts of the processes on figures 3-5, enough for the model validations are simulated. The heat transfer through the tank envelopes are not taken into account to check the possibility for its neglecting. Temperature fields and the streamlines in the water domain are shown on the next figures. Clear temperature stratification and relatively uniform temperatures at the horizontal cross sections are obvious at the case of low flow rate at the upper level of the inlet at Scenario 2 (fig. 7). In contrast the temperature stratification is almost absent at Scenario 3. Recirculating flows due to the buoyancy and the forced outflowing of the water at the bottom level are established at this case. They occupied the entire volume of the accumulator and cause unstratified temperature field in the water domain (fig. 8).

Water motions in the unused external pipe spaces are not established at the modeled scenarios, excepting the space near the flange at the bottom of the tank. So the geometrical model can be simplified by ignoring its unnecessary components with a total volume less than $2 \%$ of the volume of the tank. This will decrease the computational time and will allow flexible analyses without reducing the accuracy of the results.

Comparisons between the average temperatures at the levels of the internal thermocouples PT 100 and the temperatures, obtained at the numerical simulations at the same places are shown on figures 9-11. The maximal differences between the experimentally obtained and the computed temperatures are about $3 \%$ at Point 1 for all simulated scenarios. The possible reasons for these differences are the established errors at the upper sensors. Therefore, the models for numerical simulations of the transient processes in the water accumulators are reliable and applicable for analysis of different modes of their thermal charging.

a)

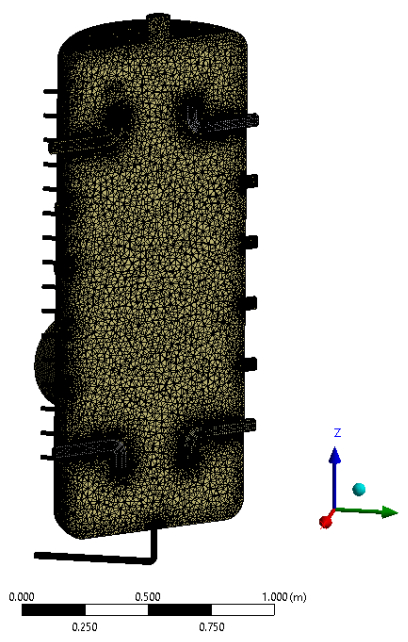

b)

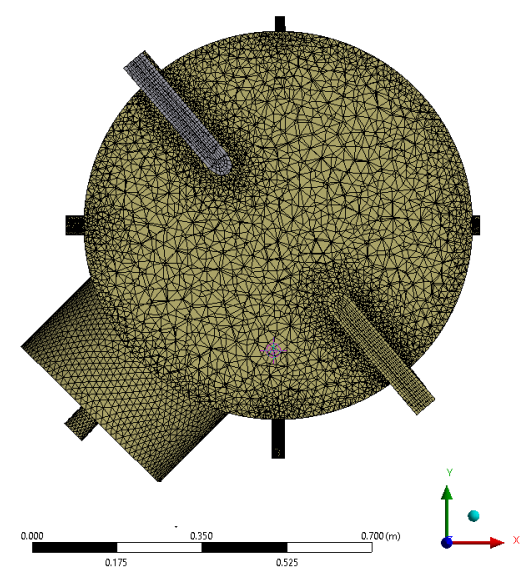

b)

Fig. 6. Finite elements mesh at vertical (a) and horizontal (b) cross-sections 


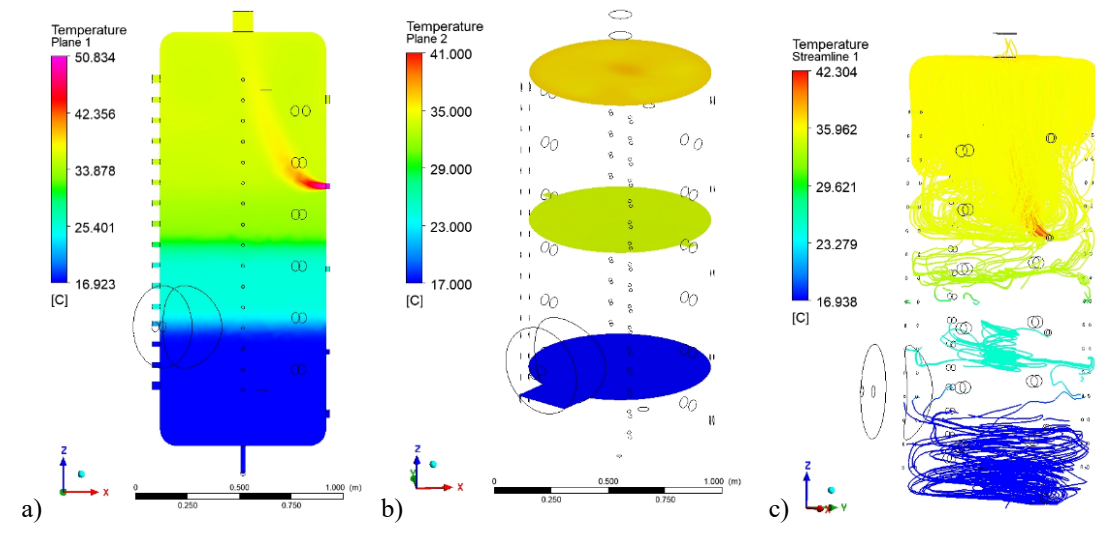

Fig. 7. Temperature fields in vertical and horizontal cross sections $(a, b)$ and streamlines (c) at a moment of Scenario 2 ( $7200 \mathrm{~s})$

Other probable reasons for the deviation of the obtained at the simulations temperatures according to the experimental data are:

- the lower turbulence in the model comparing to the real object - the platina thermocouples are fixed by metal pins in the space of the laboratory accumulator that probably caused local turbulence eddies. These pins do not present in the geometrical models.

- the neglecting of the thermal loses through the accumulator envelopes leads to higher temperature stratification in the modelled object at the lower flow rates and velocities.

Despite the mentioned inaccuracy, CFD allows detail analyses of parameters about the transient thermal and fluid flow fields in the accumulator space that cannot be obtained experimentally: streamlines, turbulence characteristics, heat transfer coefficients and other.

a)

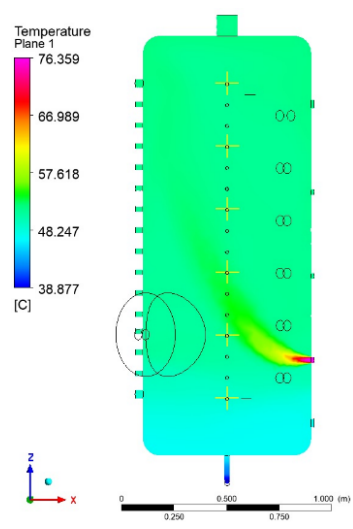

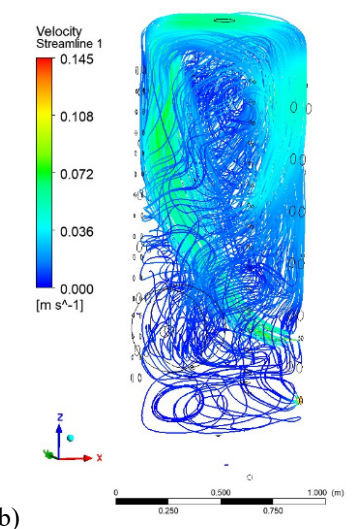

Fig. 8. Temperature fields in vertical cross section (a) and streamlines (b) at a moment of Scenario $3(3200 \mathrm{~s})$ 

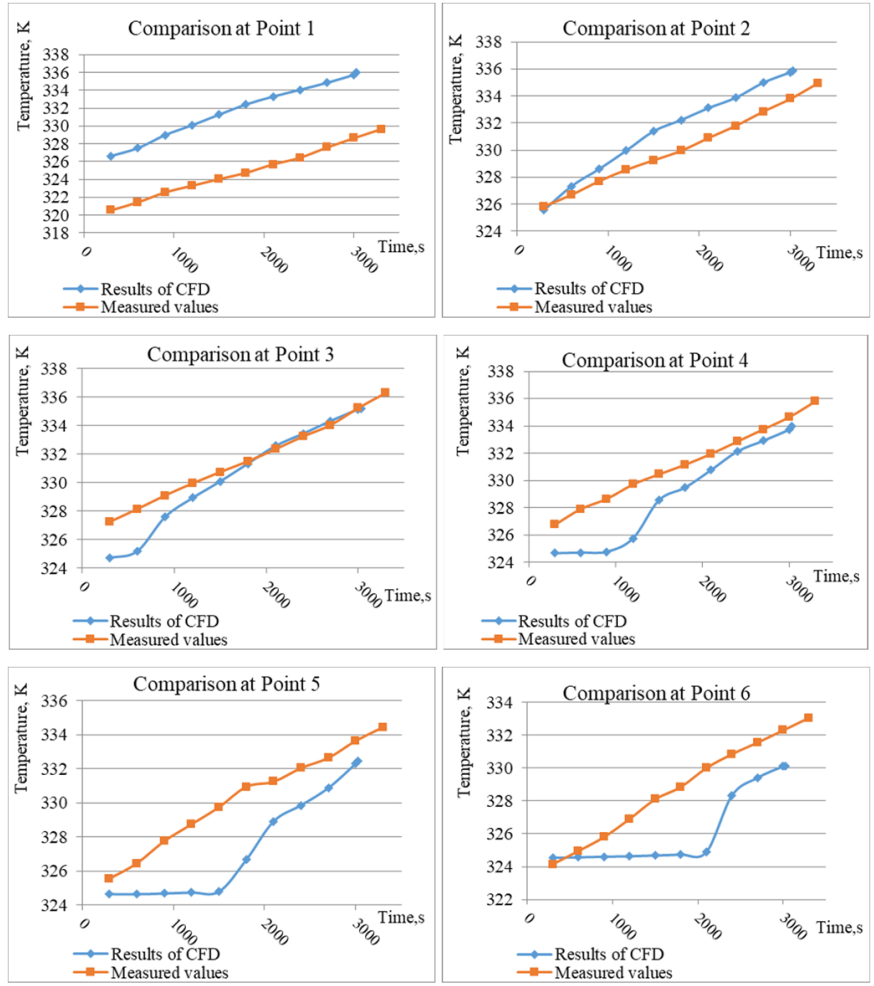

Fig. 9. Comparisons between the obtained at the measurements and the numerical simulations temperatures along the axis of the tank at Scenario 1
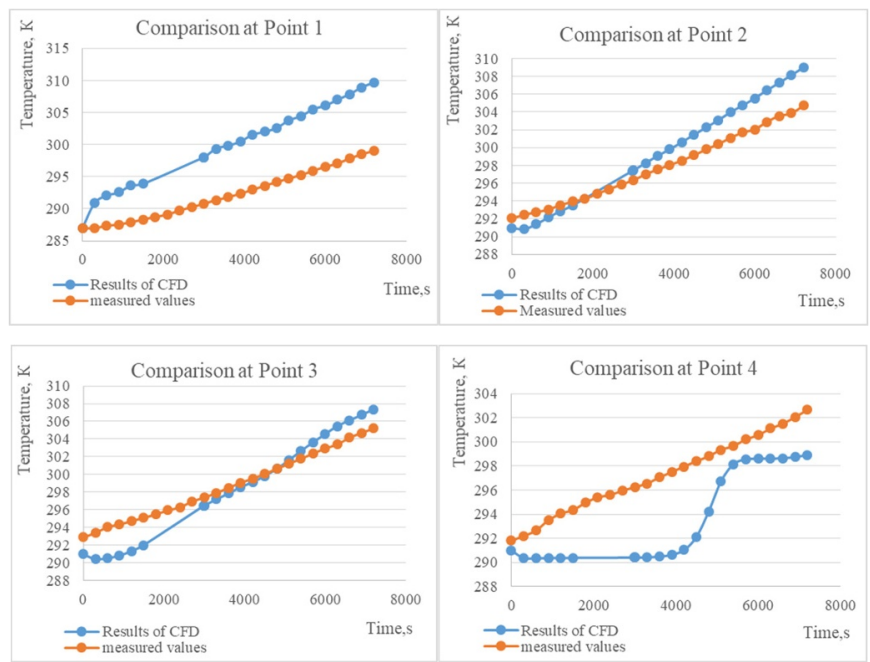


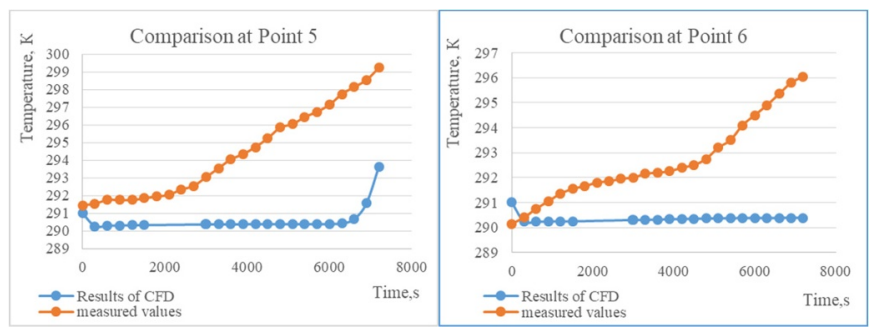

Fig. 10. Comparisons between the obtained at the measurements and the numerical simulations temperatures along the axis of the tank at Scenario 2
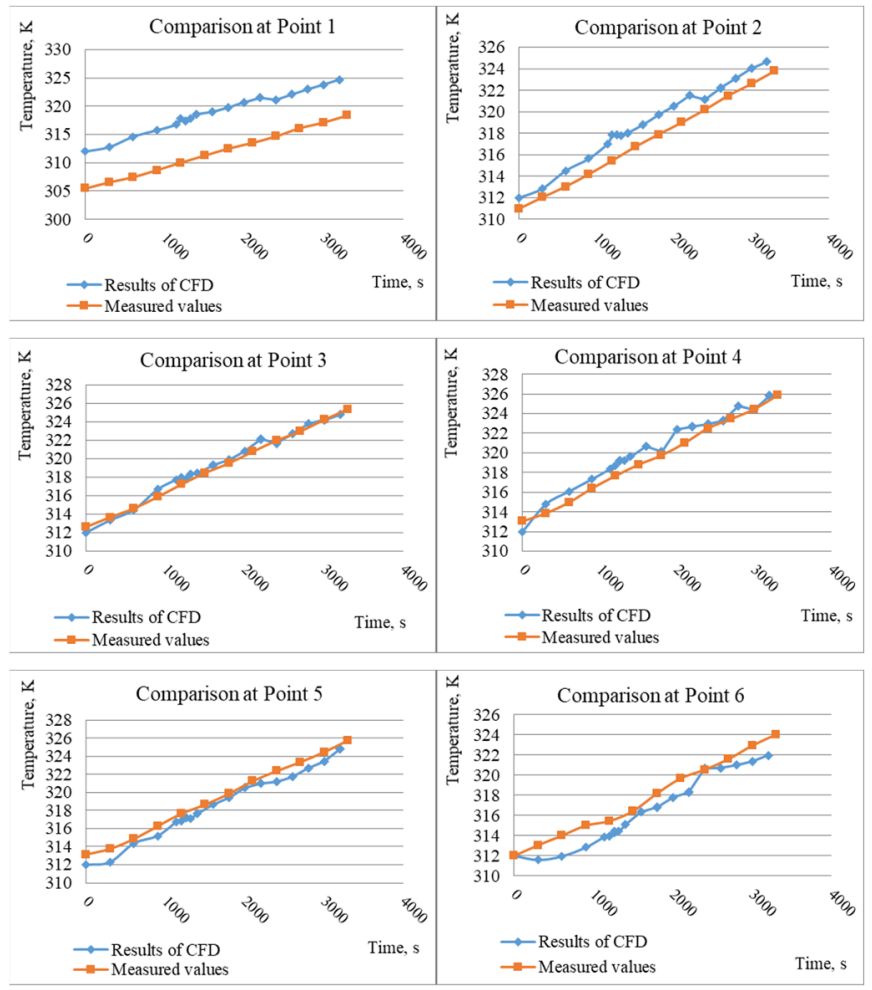

Fig. 11. Comparisons between the obtained at the measurements and the numerical simulations temperatures along the axis of the tank at Scenario 3

\section{Conclusions}

The numerical solution of the proposed mathematical model allows detailed information about the transient three-dimensional thermal and fluid flow fields in water accumulators at different configurations and charging schemes. This approach is suitable for prediction and control of the temperature stratification and the accumulated heat in order to achieve efficient 
operation mode of the thermal systems according to the characteristics of the energy sources and consumers.

Further testing and validation of the models at thermal discharging and simultaneous charging and discharging of the thermal accumulator are forthcoming. For better accuracy, it is necessary to take into account the heat transfer through the envelopes of the tank.

This study has been financially supported by the project BG05M2OP001-1.001-0008: National Centre of Mechatronics and Clean Technologies, funded by Operational Programme for Science and Education for Smart Growth at EU Funds.

\section{References}

1. Z. Wang, H. Zhang, B. Dou, H. Huang, W. Wu and Z. Wang, Renewable Energy, 111, 353-371 (2017)

2. R. I. Loehrke, M. K. Sharp, H. N. Gari and R. D. Haberstroh, Thermal Stratification Enhancement for Solar Energy Applications, Colorado State University Fort Collins Dept. of Mechanical Engineering (1977)

3. N. Kaloyanov, Analysis and synthesis of low potential combined heat systems, $\mathrm{PhD}$ Thesys, Technical Universyty of Sofia (1990)

4. C. Pagkalos, G. Dogkas, M. K. Koukou, J. Konstantaras, K. Lymperis and M. G. Vrachopoulos, International Journal of Thermofluids, 1, 100006 (2020)

5. Y. Ahmed, International Journal of Advanced Engineering and Business Sciences, 2(2), 33-50 (2021)

6. N. Penkova and N. Harryzanov, In Proceedings at 5th International Conference on Energy and Sustainability 469-477 (2014)

7. Z. Yang, H. Chen, L. Wang, Y. Sheng and Y. Wang, Renewable Energy, 85, 31-44 (2016)

8. Z. Wang, H. Zhang, B. Dou, H. Huang, W. Wu and Z. Wang, Renewable Energy, 111, 353-371 (2017)

9. T. Bouhal, S. Fertahi, Y. Agrouaz, T.El Rhafiki, T. Kousksou and A. Jamil, Numerical modeling and optimization of thermal stratification in solar hot water storage tanks for domestic applications: CFD study. Solar Energy, 157, 441-455 (2017)

10. ANSYS Release 16 - (C) SAS IP, Inc. (2016)

11. Q. Hou, and Z. Zou, ISIJ international, 45(3), 325-330 (2005)

12. R. Tsecov N. Kaloyanov and S. Chervenkov, Proceedings of 25-th International Conference of Power Engineering and Power Machines (in Bulgarian), 29-39 (2020), http://copepm.eu/documents/2020.pdf 